\title{
Hacer bien una fecha. Entre el cuidado y la maximización del placer en consumidores de drogas sintéticas
}

| ${ }^{1}$ Martín Güelman, ${ }^{2}$ Sebastián Ezequiel Sustas |

Resumen: En este artículo analizamos los procesos de vulnerabilidad y las prácticas de cuidado individuales y grupales en relación a los consumos de drogas de jóvenes que asisten a fiestas electrónicas en la Ciudad Autónoma de Buenos Aires, Argentina. Los resultados provienen de una investigación cualitativa más amplia en la que realizamos entrevistas individuales a jóvenes de entre 18 y 25 años y grupos focales que estuvieron conformados por grupos preexistentes de amigos. En contraposición a la idea de un "descontrol generalizado" y a cierta banalización del consumo de sustancias, el análisis de las experiencias de los jóvenes nos permitió observar el desarrollo de estrategias de control de los riesgos que son inherentes a estos consumos. Estas estrategias concurren, en muchas ocasiones, con la maximización de las experiencias sensoriales placenteras. Partiendo de algunos conceptos de la Salud Colectiva y la Teoría Social, analizamos las experiencias de consumo de drogas sintéticas de los jóvenes para abordar los sentidos asociados a las instancias de cuidado y las demandas por cuidado emergentes; las estrategias que emplean para la maximización del placer; y las sabidurías prácticas que despliegan y el diálogo de éstas con los conocimientos del campo de la biomedicina.

> Palabras clave: cuidados; drogas sintéticas; jóvenes; vulnerabilidad.

\footnotetext{
1 Instituto de Investigaciones Gino Germani, Universidad de Buenos Aires. Buenos Aires, Argentina (marguelman@gmail.com). ORCID: 0000-0003-4906-2336

${ }^{2}$ Instituto de Investigaciones Gino Germani, Universidad de Buenos Aires. Buenos Aires, Argentina (s.sustas@gmail.com). ORCID: 0000-0002-6215-5134
}

Recebido em: 02/01/2018 Revisado em: 04/05/2018 Aprovado em: 22/06/2018 


\section{Introducción}

Asistimos en Argentina a una reactualización del debate público sobre las consecuencias negativas para la salud vinculadas al consumo de drogas sintéticas en poblaciones juveniles. Lejos de constituir una problemática embrionaria, las Ciencias Sociales han documentado y analizado en las últimas tres décadas las formas de uso que los sujetos y grupos le dan a este tipo de sustancias, los ámbitos en los que el consumo se produce, los vínculos de sociabilidad que tienen lugar en estos ámbitos (especialmente fiestas electrónicas) y, en menor medida, las prácticas de cuidado que los propios usuarios llevan a cabo para prevenir o mitigar las derivaciones no deseadas de dichos consumos (CALAFAT FAR et al., 1998; GAMELLA; ALVAREZ ROLDÁN, 1999; CAMAROTTI, 2004; KORNBLIT, 2004; COUTINHO, 2008; CALAFAT FAR; JEREZ; DUCH, 2009; CAMAROTTI, 2010; CAMAROTTI, 2012; GÜELMAN, 2015). Kenneth Thompson (2014) señala que los profesionales que se han abocado al estudio de los riesgos asociados al consumo de éxtasis o MDMA sostienen que el problema no es tanto la sustancia química en sí misma, sino las condiciones en que se produce la ingesta de la droga durante las fiestas electrónicas o raves.

Los síntomas de la ingesta de éxtasis son casualmente similares a los de un ataque al corazón: las altas temperaturas y el baile vigoroso de las raves pueden interactuar con el MDMA [...] y producir experiencias físicas cuantitativa o cualitativamente diferentes de cuando se ingiere la droga en un estado de relajación (MERCHANDT; MACDONALD, 1994, citado en THOMPSON, 2014, p. 80-81).

En su clásico trabajo Drug, set and setting, Norman Zinberg (1984) identifica las condiciones en que se producen los consumos, es decir los entornos físicos y sociales (setting), como un elemento clave para potenciar instancias de control frente a las consecuencias no deseadas del consumo de drogas.

Por su parte, desde la óptica de quienes participan en la movida electrónica, las drogas sintéticas provocan escasos efectos secundarios. A su vez, a diferencia de otras sustancias más nocivas y con mayor potencial adictivo como la cocaína, en estas drogas sería más fácil poder controlar y elegir los momentos de consumo, lo que llevaría a los usuarios a sentir que pueden manejar la situación, sintiéndose seguros (CAMAROTTI, 2015).

La emergencia de las drogas sintéticas se produjo en Argentina en la década de 1990. En un contexto caracterizado por la acentuación del paradigma del 
consumismo y la masificación del uso de sustancias ilegalizadas (fundamentalmente cocaína y marihuana), las drogas sintéticas se erigieron como una mercancía con un fuerte atributo de diferenciación social (BOURDIEU, 1998). El éxtasis, la primera de las drogas de diseño en cobrar notoriedad, se inscribe en este contexto con un potencial de maximización del ocio y de la experiencia de nocturnidad, orientada al consumo recreativo juvenil. A diferencia de lo que ocurre con otras sustancias, el consumo de drogas sintéticas suele ser significado por los jóvenes como una práctica que solo cobra sentido en instancias recreativas y en compañía de otras personas, enmarcando así al consumo como potenciador de la sociabilidad (CAMAROTTI, 2015).

Como señala Ana Clara Camarotti (2004, p. 102):

Las características específicas del éxtasis residen en que quienes la eligen buscan estar en grupos, expuestos en espacios públicos, en raves, after, discos, fiestas privadas, conectados con otras personas que están viviendo experiencias similares, alertas a las distorsiones emocionales y sensuales que les suceden, dedicados por completo a disfrutar de ese momento de ocio y recreación, poseídos por lo que ellos denominan una fuerte y contagiosa sensación de amor, hermandad y solidaridad con las otras personas que se encuentran en el lugar.

Los estudios académicos sobre la movida electrónica o la cultura rave "[...] enfatizan la atmósfera amigable de los eventos, tipificada como un ambiente con una conducta menos agresiva, menos machista y violenta que la de la nocturnidad habitual" (THOMPSON, 2014, p. 80).

Los discursos de los jóvenes que participan de la movida electrónica, exhiben una mirada transgresora y desprejuiciada respecto de las drogas ilegalizadas que cuestiona los juicios moralizantes en torno a las mismas. Esta mirada se compone, en lo fundamental, de dos núcleos de sentido: una tajante diferenciación entre consumo y consumo problemático o bien la atribución de plena entidad al consumo recreativo; y la consideración de que el consumo de drogas no constituye una conducta desviada.

Esta mirada no supone un impedimento para que los jóvenes reconozcan los potenciales efectos negativos del consumo de drogas y emprendan acciones destinadas a prevenirlos o mitigarlos. De este modo, entre los consumidores de drogas sintéticas es posible advertir una particular propensión a la búsqueda de información sobre los componentes de las sustancias, los riesgos de su consumo y las acciones más adecuadas para paliarlos (GÜELMAN, 2015). ${ }^{1}$ Se observa también el interés por conocer y experimentar aquellas combinaciones de acciones y prácticas que permiten 
optimizar los efectos psicoactivos de las sustancias vinculados a sensaciones de bienestar. En suma, las dos dimensiones en que analíticamente pueden dividirse estas búsquedas se orientan a un objetivo que, en la práctica resulta inescindible. En otros términos, el logro $-\mathrm{y}$, especialmente, la maximización- del placer que se procuran a través del consumo de estas drogas requieren del desarrollo de estrategias de control de los riesgos que son inherentes a este consumo.

A pesar de la multiplicación de investigaciones y reflexiones alrededor de los conceptos de vulnerabilidad y cuidado desarrollados durante los últimos años desde las Ciencias Sociales de la Salud, la Medicina Social y la Salud Colectiva aún existe una vacancia de estudios en los que se articulen estas dos categorías para abordar las experiencias de jóvenes -y en particular de aquellos que en función de sus características sociodemográficas no suelen ser ubicados en los así llamados grupos de riesgo- en relación a los consumos de drogas. La fuerte inercia conceptual de asociar la vulnerabilidad a la pertenencia a un grupo de riesgo, invisibiliza problemáticas, reduce las complejidades de los fenómenos e impide observar las particularidades de cada dinámica social. Con el propósito de aportar a cubrir esta vacancia, presentamos aquí resultados provenientes de un estudio mayor cuyo objetivo general fue analizar los procesos de vulnerabilidad y las prácticas de cuidado individuales y grupales en relación a los consumos problemáticos de drogas de jóvenes de 18 a 25 años de barrios vulnerabilizados y de sectores medios que asisten a espacios recreativos nocturnos de la Ciudad Autónoma de Buenos Aires (CABA) (Argentina). Específicamente, en este artículo nos centramos en las dos dimensiones analíticas aludidas -vulnerabilidades y cuidados- para un grupo particular de jóvenes: los que asisten con frecuencia a fiestas electrónicas. Algunos de los interrogantes que guían este trabajo son: ¿cuáles son las instancias de cuidado que estos jóvenes construyen en relación a sus consumos de sustancias en fiestas electrónicas?; ¿qué vínculos se establecen entre los procesos de vulnerabilidad y las instancias de cuidado en relación a esos consumos?; ¿qué estrategias emplean para maximizar el placer que buscan a partir del consumo?; ¿de qué manera intentan compatibilizar esta búsqueda por maximizar el placer con la necesidad de minimizar los riesgos asociados al consumo?; ¿qué sabidurías prácticas despliegan y de qué manera esos saberes dialogan con los conocimientos biomédicos?; ¡¡cuáles son las demandas por cuidado de estos jóvenes?

En este marco, hacemos empleo del concepto procesos de vulnerabilidad, el cual nos permite interrelacionar las dimensiones individual, vincular y socio-institucional 
en su carácter dinámico, y romper con los reduccionismos deudores de paradigmas que asientan su fuerza explicativa en concepciones de tipo causal o lineal. El concepto de vulnerabilidad es empleado por este nuevo paradigma para visibilizar a grupos o individuos en situaciones de fragilidad social, política y/o jurídica, en la promoción, protección o garantía de sus derechos de ciudadanía, considerando que la posibilidad de enfrentarlas depende de transformaciones en las prácticas, por lo que se encuentra abierta a todos los grupos socioeconómicos (AYRES; FRANÇA JÚNIOR; JUNQUEIRA CALAZANS; SALETTI FILHO, 2008).

El carácter dinámico y procesual de nuestro objeto de estudio requiere un abordaje desde los itinerarios juveniles como categoría teórica-metodológica. El empleo de esta herramienta permite visibilizar una multiplicidad de saberes, prácticas y demandas por cuidado en las experiencias sociales y vitales. La noción de itinerario -y particularmente de itinerario terapéutico - ha sido extensamente utilizada en las últimas dos décadas en el campo de la Salud Colectiva, especialmente en Brasil, en investigaciones que han buscado brindar visibilidad y reconocimiento a la pluralidad de saberes y prácticas de cuidado de los individuos y grupos sociales. Mediante el uso de dicha categoría se ha buscado también describir los caminos recorridos por estos sujetos y grupos -sus procesos de decisión, evaluación y adherencia a tratamientos- frente a los recursos disponibles, dentro y fuera de las instituciones de salud (GERHARDT; PINHEIRO; RUIZ; GOMES DA SILVA JUNIOR, 2016).

En este artículo presentamos nuestro análisis siguiendo un recorrido por las diferentes instancias que los jóvenes atraviesan para dar forma a sus salidas recreativas nocturnas dentro de la movida electrónica. Identificamos las siguientes "estaciones" en ese recorrido: 1) el fixture; 2) la adquisición de las drogas sintéticas; 3) el testeo o la búsqueda de información sobre la composición química de las sustancias y de sus efectos psicoactivos; y 4) la ingesta de sustancias durante la fiesta.

\section{Métodos}

La investigación de la que derivan los resultados que recoge el presente artículo se situó dentro del paradigma cualitativo y, en particular, del enfoque interpretativo. Con el objeto de responder a los objetivos de la indagación, llevamos a cabo siete grupos focales con jóvenes. El universo de estudio lo conformaron jóvenes de 18 a 25 años de barrios vulnerabilizados y de sectores medios que asisten a espacios 
recreativos nocturnos de la CABA. Los casos fueron seleccionados a partir de un muestreo no probabilístico estratégico. Para conformar los grupos contactamos inicialmente a una persona y le solicitamos que reclute cinco amigos más, cuyas edades se encontraran en el rango etario establecido, con los que compartía salidas nocturnas en la CABA.

Los grupos estuvieron integrados por entre tres y seis participantes. Cuatro grupos tuvieron una composición mixta, mientras que de los tres restantes participaron únicamente varones.

Los grupos focales tuvieron una duración de entre una hora y cuarto y dos hora y veinte minutos. La moderación de los grupos focales estuvo a cargo de los autores de este artículo. Fue desarrollada una estrategia según la cual uno de los dos se encargaba de las tareas principales del moderador y la otra persona cumplía la función de asistente y observador. El moderador era el encargado de dar inicio al grupo y fijar sus alcances; describir los objetivos y el marco institucional de la investigación; dar cuenta del carácter voluntario de la participación, del anonimato y del tratamiento confidencial de la información que realizaríamos; formular la pregunta disparadora, las preguntas contenidas en la guía de pautas y aquellas vinculadas con temáticas emergentes que pudieran resultar de interés; introducir otro tipo de estímulos; desarrollar estrategias para fomentar la participación y dinamizar el debate; y establecer el momento de finalización del grupo. Por su parte, las funciones del asistente del moderador/observador eran entregar los consentimientos informados a los participantes, manejar el grabador, registrar las interacciones no verbales que no pudieran ser captadas por el grabador, describir el clima de la reunión y los aspectos contextuales del grupo focal, revisar la guía de pautas y formular preguntas que el moderador pudiera haber omitido o plantear interrogantes vinculados con temáticas emergentes pertinentes.

Pese a que cierta literatura metodológica no aconseja trabajar con grupos de amigos o de personas que se conozcan mutuamente muy bien, ya que "[...] el nivel de cosas dadas por supuestas que se mantiene implícito tiende a ser [...] alto" (FLICK, 2012, p. 134), en nuestro caso, no construimos grupos ad hoc, sino que cada grupo focal fue integrado por un grupo de amigos preexistente. Ello permitió indagar prácticas de cuidado en relación al consumo problemático de bebidas alcohólicas y drogas ilegalizadas a través de sucesos realmente acaecidos y de experiencias compartidas por los jóvenes en espacios recreativos nocturnos a 
los que asisten. Entre las ventajas de trabajar con grupos reales no construidos por el investigador se cuenta la posibilidad de aproximarse a situaciones de interacción social más "naturales" (KITZINGER, 1994).

Como afirma Betina Freidin, los grupos focales son particularmente fértiles para

[...] indagar cómo las personas en sus redes de interacción y círculos [...], optan por diferentes prácticas de cuidado, por aceptar algunas y cuestionar otras, las limitaciones que enfrentan, y los significados que otorgan a sus decisiones [...] [su] confianza en distintas modalidades de cuidado las tensiones que pueden presentarse en la vida cotidiana entre la disciplina del cuidado y el placer/disfrute/relajación, y sus percepciones diferenciales de riesgo y vulnerabilidad [...] (2016, p. 4).

El análisis de los siete grupos focales realizados nos permitió vislumbrar, en el grupo de jóvenes que asistían con frecuencia a fiestas electrónicas en la CABA, un repertorio de prácticas de cuidado que presentaba diferencias significativas respecto del resto de los grupos. Ello nos condujo a considerar que era necesario llevar a cabo un análisis pormenorizado a través de un estudio de caso de dicho grupo.

Con el fin de maximizar las posibilidades y la capacidad que las condiciones y características del caso seleccionado brindan para el desarrollo de conocimiento o la generación de teoría a partir de su estudio desarrollamos un estudio de caso instrumental. En otras palabras, en un estudio de caso instrumental, el caso puede iluminar un problema teórico-conceptual o empírico que lo trasciende. En nuestro estudio, el problema empírico que trasciende al caso seleccionado son los procesos de vulnerabilidad y las prácticas de cuidado de jóvenes en relación al consumo de drogas en espacios recreativos nocturnos.

Los jóvenes que conforman el universo de estudio que analizamos en este artículo residen en la CABA. En términos sociodemográficos, podrían clasificarse como pertenecientes a un nivel socio-económico medio con estudios universitarios en curso e inserciones laborales en empleos no manuales de mediana calificación.

El corpus de información que aquí analizamos estuvo compuesto por entrevistas individuales con los miembros del grupo y un grupo focal. Las entrevistas y el grupo focal fueron codificados con el apoyo del programa informático Atlas. ti. El manual de códigos fue construido inicialmente a partir de las dimensiones de la guía de pautas. Luego, a través de un análisis inductivo, identificamos categorías emergentes asociadas a las formas de los jóvenes de vincularse en los contextos de dichas fiestas y de comprenderse a sí mismos y a las maneras de enmarcar los consumos 
de sustancias en dichos escenarios. La identificación de las categorías emergentes se realizó en diálogo con el marco teórico y los núcleos conceptuales relevados en indagaciones empíricas precedentes. Finalmente, realizamos un análisis temático de la información construida.

En lo que refiere a las consideraciones éticas, antes de comenzar con cada grupo focal o entrevista leímos el consentimiento informado y entregamos una copia firmada a cada participante. Luego de solicitar autorización para grabar la conversación, aclaramos a los jóvenes que la transcripción, como efectivamente ocurrió, estaría a cargo del investigador y entrevistador/moderador del grupo focal, quien se encargaría de modificar los nombres de los participantes, así como de otras personas, lugares e instituciones que se mencionen, de modo tal que no puedan ser identificadas. La investigación contó con el aval del Comité de Ética del Instituto de Investigaciones Gino Germani (Facultad de Ciencias Sociales, Universidad de Buenos Aires), según resolución del 28 de octubre de 2013.

\section{Resultados}

\section{La movida electrónica}

Antes de adentrarnos en el análisis de las estrategias que los jóvenes despliegan para maximizar el placer y prevenir o mitigar las consecuencias negativas asociadas al consumo de sustancias psicoactivas, especialmente drogas sintéticas, es necesario que describamos sucintamente lo que se conoce como movida electrónica. El término fiesta electrónica es una categoría que se utiliza habitualmente para englobar una variedad de eventos que tienen en común una serie de elementos, entre los que se cuentan: los medios en que se genera la música que allí se reproduce -los sintetizadores y mezcladores que maneja el disc jockey (DJ)-; el tipo de lugares en que se desarrollan ("fechas" o festivales en boliches, estadios, espacios abiertos); y el consumo de drogas de diseño por parte de un número importante de asistentes. En las fiestas, cada DJ se inscribe en un estilo musical particular que es el eje central de la convocatoria: trance, techno, house, minimal, progressive. ${ }^{2}$ La centralidad de la música en las fiestas permite comprender las formas de sociabilidad esperadas en esos ámbitos. En otros términos, las atmósferas que los DJs generan a partir de los ritmos y cadencias son un elemento central en las dinámicas vinculares entre aquellos que son del "palo", es decir que se reconocen como integrantes de la movida electrónica. 
Camarotti (2010) identifica tres etapas del surgimiento y desarrollo de la movida electrónica en Buenos Aires. En el periodo inicial las fiestas eran cerradas y exclusivas y el éxtasis solo era consumido por un grupo reducido de concurrentes. Durante el periodo de extensión, ingresan a la escena las fiestas masivas, se intensifica la participación de los concurrentes y el éxtasis se convierte en un elemento clave. Finalmente, en el periodo de masificación la movida se consolida con la proliferación de fiestas masivas junto con eventos más selectivos y se produce una fuerte expansión del consumo de drogas sintéticas. Según la autora, en la actualidad el público que participa de la movida electrónica en Buenos Aires presenta tres características distintivas: un nivel de educación formal medio o superior; una inserción ocupacional propia de sectores socioeconómicos medios y altos; y altos grados de integración socio-afectiva.

El término "movida" tal como es utilizado por los jóvenes que participaron de nuestro estudio refiere a tres sentidos convergentes sobre conocimientos que se debe poseer: a) aspectos convocantes de la fiesta, los cuales remiten a los diferentes subgéneros electrónicos, los DJs que los ponen en escena y el reconocimiento de linajes musicales; b) prácticas vinculares esperables en el espacio donde se desarrolla la fiesta, es decir, cómo comportarse con los otros y ser aceptado o reconocido; y c) formas apropiadas de consumo de sustancias para maximizar las experiencias placenteras y minimizar la posibilidad de ocurrencia de riesgos o situaciones negativas o de displacer.

Ante el reconocimiento de que el consumo de drogas sintéticas puede acarrear consecuencias negativas de diversa índole, los jóvenes que participaron del estudio afirman sentirse menos vulnerables y más protegidos en lo que denominan "fechas" que en eventos masivos. Mientras que "las masivas", que tienen en Creamfields su representación más acabada, son fiestas que, en un mismo predio, albergan una multiplicidad de estilos de la música electrónica y cuentan con carpas o escenarios en los que se presentan una gran cantidad de DJs, las "fechas" son eventos a los que concurren pocas personas en las que predomina un subgénero musical y en las que toca un único (o unos pocos) DJs (CAMAROTTI, 2004; 2010). Las fiestas masivas no son para estos jóvenes "lugares amigables" en virtud de que a ellas asiste una gran cantidad de público que no forma parte de "la movida" y cuyos intereses para concurrir carecerían de legitimidad: únicamente para drogarse; porque está de moda; para robar; o para generar situaciones de violencia. Las características que fueron asumiendo las fiestas masivas los forzaron a ser más selectivos priorizando 
la concurrencia a aquellos lugares donde "conocen a todo el mundo" y en los que se vivencia "un espíritu más comunitario”. Las significaciones de estos jóvenes exhiben un sentimiento de nostalgia ante la difuminación de las características básicas que asumían las fiestas electrónicas en "su época", es decir, en un pasado reciente del que no se ofrece una referencia temporal precisa. Este sentimiento de nostalgia resulta inescindible de la consideración de que estos cambios se corresponden con una extendida degradación cultural que conduce a una necesaria guetización de la movida electrónica.

\section{"Hacer bien una fecha"}

En los siguientes apartados, presentaremos los resultados siguiendo un recorrido por diversas "estaciones" en el que buscaremos replicar las distintas fases que asume el "hacer bien una fecha". La expresión nativa "hacer bien una fecha" refiere al desarrollo de una serie de instancias que, según los jóvenes, es necesario respetar para lograr una experiencia satisfactoria antes, durante y después de una fiesta electrónica, en relación a la consecución de sensaciones corporales placenteras motorizadas por las sustancias, al placer que supone el encuentro sociable como tal y a la minimización de los riesgos asociados al consumo de drogas sintéticas.

El recorrido estará compuesto por cuatro postas: 1) el fixture; 2) la adquisición de las drogas sintéticas; 3) el testeo o la búsqueda de información sobre la composición química de las sustancias y de sus efectos psicoactivos; y 4) la ingesta de sustancias durante la fiesta.

\section{Primera parada: el fixture}

En la jerga deportiva, el término fixture refiere a un cronograma con la sucesión de partidos que debe disputar un equipo en un campeonato. La alusión al término fixture o schedule en el contexto que analizamos remite a una serie de instancias de elección y selección de "fechas" de DJs en un período de tiempo dado. Esta planificación consiste en la delimitación de todos los eventos o presentaciones de DJs que les resultan de interés y, de ser posible, la adquisición de entradas anticipadas. Cuando esto último no resulta posible por restricciones económicas, los jóvenes intentan separar el dinero cada mes para luego llevar a cabo la compra de manera colectiva. La asistencia a la fechas puede encontrar en el DJ y el estilo musical el aglutinador 
principal, pero también es la ocasión para transitar por determinados espacios de sociabilidad, juntarse con amigos y consumir algunas sustancias de forma recreativa.

Los participantes señalan que si saben que no pueden hacer bien una fecha, es decir, reunir el "combo completo" que ello supone -incluyendo la adquisición de las drogas sintéticas- prefieren no asistir al evento. Ante la restricción de dinero y frente a la disyuntiva de asistir a más fiestas o concurrir a menos fechas pero "haciendo bien" cada una de ellas, siempre optan por esta última alternativa.

\section{Segunda parada: la adquisición de las drogas sintéticas}

La masificación de la movida electrónica conlleva para los jóvenes una serie de corolarios negativos entre los que se cuenta un significativo deterioro de la calidad de las drogas sintéticas que se comercializan ilegalmente. El aumento en la demanda fruto de esta masificación habría generado un aumento en la oferta y en las modalidades de adulteración de drogas. Aprovechando la homogeneidad de las pastillas en su aspecto exterior, los vendedores, en ocasiones, entregan fármacos en lugar de sustancias psicoactivas o las adulteran con componentes altamente nocivos para la salud. La constatación de este fenómeno constituye el argumento central de estos jóvenes no solo para no comprar drogas a vendedores desconocidos o en las propias fiestas, sino también, como veremos en la siguiente parada, para llevar adelante estrategias que permitan conocer la composición química de las drogas. La posibilidad de comprar pastillas a un "dealer" del que no tienen referencia, o bien, dentro de las propias fiestas electrónicas (aun si se agotaron las dosis que llevaban y tienen voluntad de consumir más) está absolutamente vedada. Esta regla inquebrantable adquiere mayor relevancia ante la constatación de que en la actualidad "hay mucha porquería dando vuelta".

\section{Tercera parada: reviews y testeo de las pastillas}

La masificación de la movida electrónica halla en los medios de comunicación un sentido asociado a cierta banalización de los consumos que se acentúa con la mediatización de fiestas o festivales en que ocurrieron muertes de jóvenes vinculadas al uso de drogas de diseño. ${ }^{3}$ En este contexto, y sobre este trasfondo, también es posible entrever ajustes que refieren a algunas prácticas de los jóvenes que componen nuestro universo de estudio. 


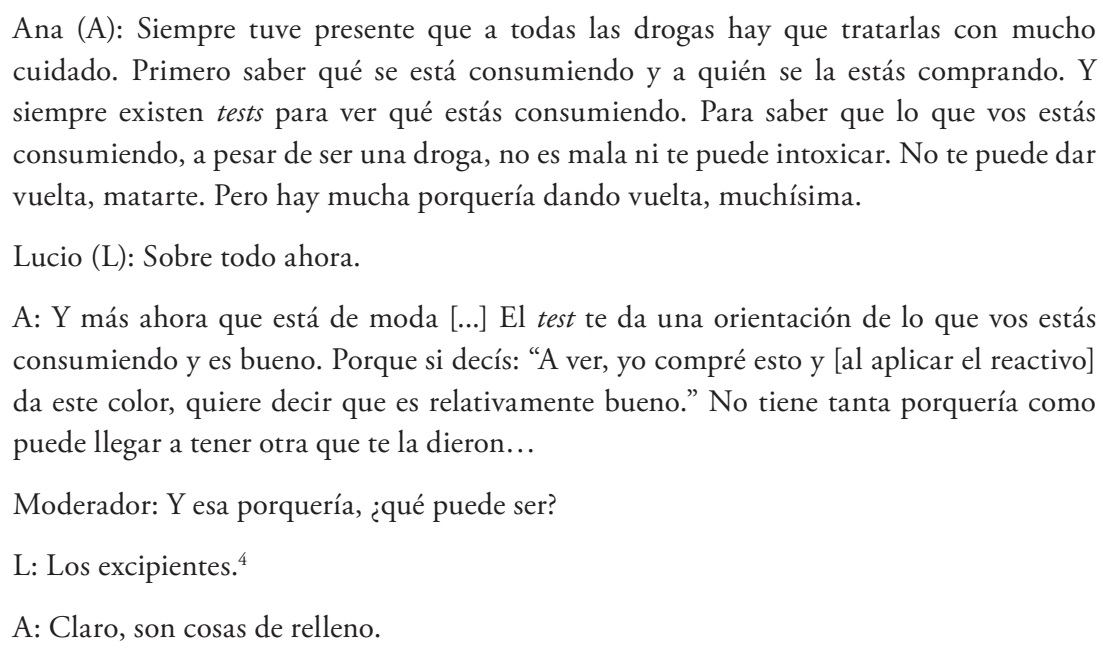

Como observamos en el fragmento del grupo focal precedente y veremos en el verbatim de entrevista a continuación, el contexto de masificación de la movida electrónica, aumento de la oferta de drogas sintéticas y degradación de su calidad, brinda contornos particulares a la importancia de conocer qué es lo que se está consumiendo. Para ello, los jóvenes desarrollan dos estrategias. La herramienta más efectiva que emplean para conocer con precisión la composición química de las drogas sintéticas es el “Test de Marquis” (PLOTKIN, 2004).

[El Test de Marquis] es un test que se vende, lo vende un usuario [de drogas de diseño], es un reactivo en el que vos tomás una muestra de la pastilla que compraste y te fijás cómo reacciona, mayormente te podés dar cuenta qué tiene, qué no tiene [...] Vos lo comprás y comprás el reactivo y te viene con el frasquito y con un instructivo donde te dice si el reactivo tiene este color tiene esto, si sale bien negro se puede tomar porque es $\mathrm{MD}[\mathrm{MA}]$, si sale amarillo [...], es catinona. Si sale de otro color no tomés nada porque puede ser piperacina, pueden ser sustancias que vos no conocés lo que tiene, entonces no la tomes. La comprás jjodete! Estás comprando algo ilegal, no sabes de dónde viene, no tiene prospecto, no tiene nada [...] (Gastón).

Cuando no pueden acceder a un Test de Marquis o bien cuando éste arroja resultados indeterminados, los jóvenes recurren a blogs de Internet especializados en la temática en los que es posible acceder a reviews: relatos de los propios consumidores sobre sus experiencias con determinada droga sintética, la composición de cada pastilla y los efectos vivenciados (SÁNCHEZ ANTELO; MÉNDES DIZ, 2015). La disponibilidad de información habilita para los jóvenes la posibilidad de llevar a cabo "un consumo responsable" que relativiza los riesgos que resultan inherentes 
a estas drogas en virtud de su carácter ilegal y la consiguiente ignorancia sobre su procedencia y su proceso de elaboración.

El conocimiento de los efectos esperables asociados a cada sustancia es el paso posterior a la identificación de su composición química. El relevamiento de información, incluyendo la aplicación del Test de Marquis, posibilita la comprobación esperable de las composiciones químicas que permiten la maximización y potenciación de las sensaciones esperadas que son disparadas por el consumo.

Los lenguajes que se utilizan para la construcción de un relato de las afecciones producidas por la conjunción de los elementos que participan de los consumos remiten al campo psicológico. De manera difusa, emergen también referencias a una interacción de otro orden que tienen su locus a nivel cerebral y un vocabulario que encuentra sentido a nivel químico. Durante los encuentros con los participantes, surgieron, en varias ocasiones, referencias a las composiciones químicas de las sustancias, sus efectos en clave neurológica y su vinculación con las sensaciones placenteras buscadas.

[El éxtasis] [...] ataca directamente en los receptores de serotonina, entonces lo que hace es liberar toda la serotonina que tiene el cuerpo, o fuerza al cerebro a conectar con serotonina de los receptores. Entonces, justamente esos receptores son los que generan sensación de bienestar (Gastón).

Estas alusiones a elementos de las neurociencias nos llevan a preguntarnos por los saberes que son empleados por los jóvenes para describir sus prácticas, sus entornos y a sí mismos. En el caso que analizamos, lo emergente está dado por el empleo de discursos que encuentran su fuente de legitimidad en saberes biomédicos de emergencia relativamente reciente (MANTILLA, 2015).

\section{Cuarta parada: la ingesta}

La cuarta parada de este periplo es el momento en que acontece el consumo. La dosificación de la ingesta a lo largo de la fecha supone, una vez más, la confluencia de dos facetas de un mismo proceso: no traspasar los umbrales de cuidado establecidos y lograr una maximización de las sensaciones placenteras que se buscan a través del consumo. La dosificación, como técnica de medición química, alude tanto a los miligramos consumidos, como a los intervalos de los efectos. Dentro del grupo de pares, el conocimiento del "experto" o ya iniciado en las sensaciones esperadas derivadas del consumo de determinado tipo de droga sintética es transmitido al novato 
tanto de manera "teórica" como ex post facto, especialmente luego de la ocurrencia de sucesos desagradables. Ello va dando lugar a un proceso de expertización respecto de las sustancias que permite encuadrar, dentro de las experiencias sensoriales, aquellas que son producidas por el consumo. Si bien coincidimos con María Epele (2015) en que el proceso de expertización surge de los propios saberes institucionales, consideramos que implica también la potencialidad de apropiación por parte de los sujetos de esos mismos saberes para actuar de manera diferente. En breve, entendemos que este proceso habilita las posibilidades de agencia.

El proceso de expertización permite a su vez conocer cuántas pastillas puede uno consumir durante la fiesta y cuánto tiempo debe dejar transcurrir entre una dosis y la siguiente.

Gastón $(\mathrm{G}):[\ldots]$ creo que fue la segunda vez que consumí, consumí de más...en los tiempos que uno tendría que conocerse a uno mismo... y yo tomé media [pastilla] de una muy fuerte y también la otra media la tomé dentro del lapso de una hora. Entonces, es como que me hizo efecto las dos medias y yo no conocía el efecto de la subida. Entonces subió muy rápido y yo pensé que me bajaba la presión o algo que no podía controlar y justamente era el efecto de la droga, que yo no me lo esperaba. Entonces me sacaron del boliche, me sentaron, me pusieron hielo...

Moderador: ¿Quién te sacó?

G: Una amiga que ya conocía los efectos y todo y me dijo "no, boludo, estás drogado, eso es lo que pasa, date cuenta" o sea, "uy, pero yo no quería esto y otro".

Lucio (L): De hecho, hemos llevado y nos ha sobrado. No es que decís: "Bueno, me llevo ocho [pastillas] y nos tomamos las ocho". Se llega a un límite. Decís: "Bueno, yo hasta acá llego porque estoy joya [muy bien]”. Y ahí queda. No es que fisurás y te clavás [tomás] media más, y a la media, la otra. O sea, nosotros nos manejamos así. No sé el resto.

Ana (A): Además a veces se ponen ciertos horarios [límites para consumir] también. Tampoco llegar [a tu casa] y estar mirando el techo hasta las 3 de la tarde [porque uno no se puede dormir por los efectos de la droga].

L: Si ves que te pegó y pasó una hora y no la necesitás...

A: No la tomás. O sea, nosotros nos manejamos así. Hay mucha gente que no. Quizás a los 20 minutos esa una entera que se tomó porque no la dejó [hacer el efecto] y se metió otra y, qué sé yo, a la media hora la tenés vomitando o lo tenés...ido.

Un momento clave del proceso se produce cuando la sustancia consumida "sube", es decir, cuando comienzan a desplegarse sus efectos psicoactivos. Los jóvenes señalan que, de este proceso y en función de la acción conjunta de una serie de condicionantes, emergen dos tipos de experiencias contrapuestas: "malviajar" y 
"bienviajar". El "buenviaje" concentra menor cantidad de menciones y reflexiones que el "malviaje" y puede definirse como la consecución de los efectos buscados a través del consumo en los que las diversas instancias de placer resultan maximizadas. Por su parte, el "malviaje" supone una "sensación de incomodidad con el lugar, la gente, la música, el ambiente que se pone muy tenso, muy oscuro", en el que la persona "deja de bailar y tiene cara de susto". "Por más que sea el DJ que amás, si la rola [la pastilla de éxtasis] te pegó mal decís: 'Loco, no me gusta [la música]' (Ana)”. $\mathrm{Al}$ indagar los motivos que pueden generar el "malviaje", las significaciones de los jóvenes se direccionaron explícitamente a los campos mental y psicológico:

Moderador: ¿Y te pega mal porque te pasás con la dosis o te puede pegar mal porque...?
Ana: Al ser psicológico, yo siempre tengo la pauta de estar bien para consumir. No estar
deprimida, no estar enojada, no tener ninguna preocupación muy fuerte, porque al ser
psicológico te trae todas esas cosas. Entonces capaz que vos viste algo que te hizo acordar
a eso y ya está. Tu cabeza se va a meter en eso y al estar enroscadísimo empezás a pensar.
Primero estar bien uno mismo, psicológicamente por lo menos. Gastón: Podés “malviajar”, porque es hiperpsicológico y estás súper sensitivo.

En su clásico estudio sobre los fumadores de marihuana, Howard Becker ([1963] 2009) señala que para que un consumo recreativo tenga continuidad en el tiempo es necesaria una conexión que lo vincule al placer. ${ }^{5}$ Para que el sujeto decida seguir incursionando en el consumo es necesario que deje atrás la ansiedad vinculada con su falta de familiaridad con los efectos de las drogas y resignifique aquellas sensaciones desagradables que puede haber vivenciado en ocasiones previas de consumo (ZINBERG, 1984). La continuidad en el consumo exige una expertización que permita discriminar las experiencias displacenteras que podrían englobarse dentro de lo que se define como un "malviaje" de los prolegómenos necesarios de un "buenviaje" que, en los primeros contactos con estas sustancias, pueden revestir un carácter indeterminado. ${ }^{6}$ Como resaltan Victoria Inés María Sánchez Antelo y Ana María Méndes Diz (2015), el "malviaje" no suele conducir al abandono del consumo, sino a su regulación y a la búsqueda por hacerse experto.

En ese momento paranoiqueé, me cagué hasta las patas [me asusté] porque entré a tomar agua de la sensación de malestar que no conocía. Hoy te puedo decir que esa sensación era de una buena rola que subía bien y yo no conocía el efecto y me pegó a malviajar. Pero era una buena rola, la iba a pasar bien. Hoy si vuelvo a sentir ese efecto sé cómo viene la mano. Sé que viene bien, no que viene mal (Gastón). 
La maximización del placer asociado a la ingesta de este tipo de sustancias supone también la confluencia de un conjunto de elementos periféricos que "acompañan el consumo". Los "ambientes" o "atmósferas" en que se enmarcan los consumos no son meras locaciones. Las características de los espacios de sociabilidad (la música, el local donde transcurre la fiesta, las personas que circulan por dichos ámbitos) predisponen de diversas maneras a quienes van a consumir. Junto con ello, resulta fundamental haberse alimentado bien previamente y acompañar el consumo con la ingesta frecuente de agua para evitar la hipertermia y la deshidratación que provocan las pastillas y con chicles, caramelos u otros dulces para "levantar la glucosa y evitar bajones de presión”. Estrategias simples como el consumo de agua y dulces, o trasladar a quien presenta un "malviaje" ("sacar") a un sitio con menos cantidad de gente, con mejor circulación de aire y con niveles de música más bajos, exponen la centralidad del entorno físico y social (setting) para lograr el disfrute de la experiencia sensorial en un contexto de relativo control de los consumos. Esto no significa que las propiedades farmacológicas de cada sustancia o la personalidad o actitudes del usuario no tengan importancia. Es necesario entender, en cada caso, como las características específicas de la droga y la personalidad del consumidor interactúan con y son modificadas por el setting y sus controles (ZINBERG, 1984).

Las distintas instancias constitutivas del consumo que, en términos analíticos, distinguimos en cuatro "paradas", implican una búsqueda consciente por maximizar el placer asociado al uso de drogas sintéticas y minimizar los riesgos. Sin embargo, la estrategia de "previsión total" encuentra límites objetivos. Frente a un grado de incertidumbre del que no es posible escapar, el consumo de este tipo de sustancias implica siempre un "salto de fe".

Entrevistador: ¿Y pensás que estas experiencias buenas [que tuviste en relación con el consumo] se explican por todas estas medidas?

Gastón: ¿̨Por todas las medidas que tomé antes de...? No, no, en realidad las medidas son para ver si la tomo o no [a la pastilla]. Cuando la tomo, ahí ya empieza a jugar mi cabeza y cómo me voy a controlar y cómo me va a pegar a mí. Siempre respeto y no sabés cómo te va a pegar.

\section{Reflexiones finales}

El cuidado ha sido equiparado históricamente al cuidado médico e institucional. Las prácticas de autocuidado han quedado relegadas, en la investigación eintervención 
sanitaria, a un segundo plano, pese a su preeminencia en la vida cotidiana de los sujetos (SÁNCHEZ ANTELO; MÉNDES DIZ, 2015). El concepto de cuidado en salud, como señala Roseni Pinheiro (2007; 2010), tiende a estar subordinado a normatividades técnicas validadas por saberes y poderes biomédicos hegemónicos, contribuyendo a la reproducción de una concepción empobrecida y reificada de las identidades. En el caso de los cuidados en salud en la población joven y adolescente, y sobre todo en relación a los consumos de sustancias, esta perspectiva concurre con visiones que negativizan las prácticas de dichos grupos etarios (CHAVES, 2010). En conjunto, esta situación tiende a profundizar las barreras tanto epistemológicas como, fundamentalmente, político-simbólicas, que obstaculizan las transformaciones de las prácticas cotidianas y de las relaciones entre los diversos agentes y los usuarios en el campo de la salud pública. A partir de esta ruptura teórico-práctica, es posible reconstruir una definición de la categoría de cuidado que integre los diversos tipos de sabidurías prácticas y de acciones dialógicas cotidianas. Desde esta redefinición conceptual, la categoría trasciende el campo biomédico y permite visibilizar las prácticas cotidianas que confluyen con el cuidado, así como las experiencias que expresan demandas por cuidado (PINHEIRO, 2010).

Así, la puesta en escena de toda una gama de prácticas que enmarcan los consumos de drogas sintéticas en vistas a la maximización de las experiencias sensoriales placenteras pareciera contrarrestar, en parte, el sentido común construido en torno a los consumos de estas poblaciones. Lejos de la inexistencia de prácticas de cuidado, se presenta toda una serie de instancias que se contraponen a la idea de un "descontrol generalizado" y una banalización del consumo de sustancias, fundamentalmente drogas sintéticas. Estas instancias se enmarcan en un conjunto de prácticas donde convergen dimensiones que, en una lectura superficial, parecieran contradictorias: la maximización del placer del consumo y el desarrollo de estrategias variadas de control de los riesgos. Sin embargo, en la conjunción de elementos que, en apariencia, son de naturaleza disímil tal vez puedan encontrarse las claves para comprender los gradientes diferenciales de los procesos de vulnerabilidad a que se enfrentan los jóvenes en relación a los consumos de drogas sintéticas. Ambas instancias requieren comprender las temporalidades y espacialidades donde se centran los consumos: los escenarios donde se enmarcan y los itinerarios de los jóvenes.

A partir de las experiencias de los jóvenes en relación a los consumos de sustancias en la movida electrónica, nos fue posible observar una multiplicidad de saberes y 
prácticas en torno al cuidado, que dialogan con los saberes biomédicos hegemónicos, pero también los tensionan y trascienden. Asimismo, en esos itinerarios juveniles se expone la impronta por demandas en torno al cuidado, sobre todo en la denuncia, más o menos velada, de los obstáculos que conlleva el carácter ilegalizado de las sustancias para la realización de tests que den cuenta de las composiciones químicas de las pastillas.

Un elemento que se presenta con fuerza en los relatos es la centralidad del grupo de pares, no sólo en relación al ámbito de sociabilidad en que se enmarcan los consumos, sino también respecto de la transmisión y construcción de saberes y prácticas de cuidado (ZINBERG, 1984). Las sabidurías prácticas en relación a los cuidados conllevan un proceso de expertización que es construido desde los saberes hegemónicos al tiempo que habilita una serie de instancias en torno a la reflexividad, la autonomía y la auto-afirmación que redundan en prácticas más saludables en relación a los consumos de drogas sintéticas. De alguna forma, podríamos sugerir cierta configuración en clave de pluralismos terapéuticos o, en términos de Zinberg (1984), elementos que constituyen una perspectiva de control de los consumos realizados por los propios usuarios.

Finalmente, otra dimensión emergente es el trabajo de los jóvenes por dar coherencia, en sus experiencias, a dos tendencias que se presentan con fuerte regularidad en esta etapa vital de sus vidas: el imperativo del disfrute y el imperativo de los cuidados y la vida saludable (SÁNCHEZ ANTELO, 2015). Estas dos lógicas, en ocasiones, presentan tensiones pero los jóvenes, en sus experiencias recreativas, buscan conciliarlas de modo tal de "hacer bien una fecha".

\section{Referencias}

AYRES, J. R. C. M. et al. El concepto de vulnerabilidad y las prácticas de salud: nuevas perspectivas y desafíos. In: CZERESNIA, D.; MACHADO DE FREITAS, C. (Eds.). Promoción de la salud. Conceptos, reflexiones y tendencias. Buenos Aires: Ediciones Lugar, 2008. p. 135-161.

BECKER, H. Outsiders. Hacia una sociología de la desviación. Buenos Aires: Siglo XXI, [1963] 2009.

BELTRAMINO, F. Música y droga: la rave como fenómeno socioestético. In: KORNBLIT, A. L. (Ed.). Nuevos estudios sobre drogadicción en la Argentina: consumo e identidad. Buenos Aires: Biblos, 2004. p. 116-125. 
BOURDIEU, P. La distinción. Criterio y bases sociales del gusto. Madrid: Taurus, 1998.

CALAFAT FAR, A. et al. Risk and control in the recreational drug culture. Palma de Mallorca: IREFREA, 1998.

CALAFAT FAR, A.; JEREZ, M. J.; DUCH, M. A. Intervenciones preventivas en contextos recreativos nocturnos: revisión. Adicciones: Revista de Socidrogalcohol, v. 21, p. 387-414, 2009.

CAMAROTTI, A. C. Consumo recreativo de drogas en jóvenes: una puesta al día. In: KORNBLIT, A. L. (Ed.). Nuevos estudios sobre drogadicción en la Argentina: consumo e identidad. Buenos Aires: Biblos, 2004. p. 101-115.

- Prácticas, discursos y nuevos espacios de sociabilidad en torno al consumo de éxtasis de jóvenes de sectores medios de la Ciudad de Buenos Aires. 2010. Tesis (Doctorado en Ciencias Sociales)-Facultad de Ciencias Sociales, Universidad de Buenos Aires, Buenos Aires, 2010.

Experiencias comunitarias de jóvenes: el circuito de música electrónica de la Ciudad de Buenos Aires. In: KORNBLIT, A. L.; CAMAROTTI, A. C.; WALD G. (Eds.). Salud, sociedady derechos: investigaciones y debates interdisciplinarios. Buenos Aires: Teseo, 2012. p. 341-360.

Experiencias comunitarias de cuidado y diversión en circuitos de música electrónica. In: DI LEO, P. F.; CAMAROTTI, A. C. (Eds.). Individuación y reconocimiento. Experiencias de jóvenes en la sociedad actual. Buenos Aires: Teseo, 2015. p. 143-170.

COUTINHO, T. O uso do corpo nos festivais de música eletrônica. In: CAIUBY LABATE, B.; GOULART, S. L.; FIORE, M.; MACRAE, E.; CARNEIRO, H. (Eds.). Drogas e cultura: novas perspectivas. Salvador de Bahía: Edufba, 2008. p. 411-431.

CHAVES, M. Jóvenes, territorios y complicidades. Una antropología de la juventud urbana. Buenos Aires: Espacio Editorial, 2010.

FLICK, U. Introducción a la investigación cualitativa. Madrid: Ediciones Morata, 2012.

FREIDIN, B. Revisando el uso de grupos focalizados en la investigación social. Revista Latinoamericana de Metodología de la Investigación Social, v. 6, 2016.

GAMELLA, J. F.; ALVAREZ ROLDÁN, A. Las rutas del éxtasis. Drogas de síntesis y nuevas culturas juveniles. Barcelona: Editorial Ariel, 1999.

GERHARDT, T. E. et al. A. Itinerários terapêuticos: integralidade no cuidado, avaliação e formação em saúde. Rio de Janeiro: Cepesc, 2016.

GÜELMAN, M. "Entre nosotros nos cuidamos siempre": consumos de drogas y prácticas de cuidado en espacios recreativos nocturnos. In: DI LEO, P. F.; CAMAROTTI, A. C. (Eds.). Individuación y reconocimiento. Experiencias de jóvenes en la sociedad actual. Buenos Aires: Teseo, 2015. p. 143-170. 
KITZINGER, J. The methodology of Focus Groups: the importance of interaction between research participants. Sociology of Health and Illness, v. 16, p. 103-121, 1994.

KORNBLIT, A. L. Nuevos estudios sobre drogadicción en la Argentina: consumo e identidad. Buenos Aires: Biblos, 2004.

MANTILLA, M. J. Educating "cerebral subjects": the emergence of brain talk in the Argentinean society. BioSocieties, v. 10, p. 84-106, 2015.

PINHEIRO, R. Cuidado como um valor: um ensaio sobre o (re)pensar a açao na construçao de prácticas eficaces de integralidade em saúde. In: PINHEIRO, R.; MATTOS, A. C. (Eds.). Razóes públicas para a integralidade em saúde: o cuidado como valor. Río de Janeiro: Cepesc, 2007. p. 15-28.

PINHEIRO, R. Demanda por cuidado como direito humano á saúde: um ensaio teóricoprático sobre o cuidado como valor dos valores. In: PINHEIRO; SILVA JUNIOR, A. G. (Eds.). Por uma sociedade cuidadora. Rio de Janeiro: Cepesc, 2010. p. 17-37.

PLOTKIN, P. El enigma del éxtasis. Rolling Stone, Buenos Aires, 2004.

THOMPSON, K. Pánicos morales acerca de la juventud. In: . Pánicos morales. Bernal: Universidad Nacional de Quilmes, 2014. p. 67-82.

ZINBERG, N. Drug, set and setting. The Basis for Controlled Intoxicant Use. New Haven: Yale University Press, 1984.

\section{Agradecimientos}

Agradecemos a la Comisión Nacional Salud Investiga del Ministerio de Salud de la Nación Argentina por financiar la investigación.

\footnotetext{
Notas

${ }^{1}$ Esta búsqueda no es exclusiva de los usuarios de drogas sintéticas, sino que también puede reconocerse, por ejemplo, entre quienes forman parte de la "cultura cannábica".

${ }^{2}$ Para profundizar en aspectos vinculados a los estilos musicales y estéticos de las fiestas electrónicas, sugerimos consultar el trabajo de Fabián Beltramino (2004).

${ }^{3}$ El 16 de abril de 2016 se realizó en la CABA la fiesta Time Warp. Durante el evento murieron cinco jóvenes producto de irregularidades en la organización (falta de ventilación, baños y puntos de hidratación insuficientes, sobreventa de tickets), ausencia de controles por parte de las autoridades municipales, connivencia del personal de la Prefectura Naval Argentina y presencia de dealers que vendían drogas sintéticas, algunas de ellas adulteradas.

${ }^{4}$ Los excipientes son componentes farmacológicos que se ańaden a la sustancia activa con el propósito de darle forma, posibilitar su conservación y vehiculizar que el principio activo de la droga ejerza el efecto deseado. En general, se busca que los excipientes sean inertes.
} 
${ }^{5}$ Vale resaltar que el libro de Becker fue publicado originalmente antes de la emergencia del fenómeno que analizamos en este artículo y aborda una modalidad de consumo que presenta diferencias significativas respecto del uso de drogas sintéticas. Sin embargo, el modelo analítico que desarrolla nos resulta fructífero para comprender la significación y resignificación de las sensaciones corporales atribuidas a las sustancias que llevan a cabo los consumidores y las vinculaciones que establecen entre dichas sensaciones y el sostenimiento en el tiempo del consumo recreativo.

${ }^{6}$ Esta expertización debe ser interpretada a la luz del proceso de aprendizaje social sobre los efectos esperados del consumo de una sustancia determinada. Este aprendizaje social lleva a que la experiencia de consumo vaya sufriendo modificaciones con el paso del tiempo. A modo de ejemplo, quien consumía sustancias psicodélicas en la década de 1960 carecía de un horizonte claro respecto de los efectos potenciales que dicho uso desencadenaría, por lo que la experiencia era más extrema. En el decenio siguiente, las personas ya habían estado expuestas a estímulos psicodélicos de diversa índole (música, colores, sensaciones, etc.), por lo que se encontraban más preparadas no solo para afrontar la experiencia, sino para hacerlo con mayores controles (ZINBERG, 1984).

${ }^{7}$ M. Güelman y S. E. Sustas participaron en el desarrollo del trabajo de campo, analizaron los datos y redactaron el manuscrito. 


\section{Abstract}

\section{Doing right the fest. Between care and maximization of pleasure in synthetic drugs users}

In this article we analyze the vulnerability processes and the individual and group care practices related to drug consumption on young people that go to electronic music festivals in Buenos Aires, Argentina. The findings come from a wider qualitative research project. For the construction of the empirical evidence, we interviewed young people from 18 to 25 years and we conducted focus groups with preexistent groups of friends that hang out together. In opposition to the idea of a generalized lack of control and a certain banalization of drug use, the analysis of young people experiences showed how they develop strategies to control the risks that are inherent to this drug use. These strategies frequently go together with the maximization of pleasurable sensorial experiences. Employing some theoretical concepts from the Collective Health and the Social Theory, we analyze the experiences of synthetic drug use by young people to approach the meanings that are associated with care practices and the emerging demands for care; the strategies that they employ to maximize the pleasure; and the practical knowledge that they develop and its dialogue with the knowledge that comes from the field of the biomedicine.

> Keywords: Care; synthetic drugs, vulnerability; young people. 


\section{Resumo}

Fazendo a festa. Entre o cuidado e a maximização do prazer em usuários de drogas sintéticas

Neste artigo analisamos os processos de vulnerabilidade e as práticas de cuidado individual e grupal relacionados ao consumo de drogas em jovens que frequentam festivais de música eletrônica em Buenos Aires, Argentina. Os resultados vêm de um projeto de pesquisa qualitativa mais amplo. Para a construção da evidência empírica, entrevistamos jovens de 18 a 25 anos e conduzimos grupos focais com grupos preexistentes de amigos que saem juntos. Em oposição à ideia de um descontrole generalizado e de certa banalização do uso de drogas, a análise das experiências dos jovens mostrou como eles desenvolvem estratégias para controlar os riscos inerentes a esse uso de drogas. Essas estratégias frequentemente acompanham a maximização de experiências sensoriais prazerosas. Empregando alguns conceitos teóricos da Saúde Coletiva e da Teoria Social, analisamos as experiências do uso de drogas sintéticas pelos jovens para abordar os significados que estão associados às práticas de cuidado $\mathrm{e}$ às demandas emergentes por cuidado; as estratégias que eles empregam para maximizar o prazer; e o conhecimento prático que desenvolvem e o diálogo com o conhecimento que vem do campo da biomedicina.

Palavras-chave: cuidado; drogas sintéticas; vulnerabilidade; jovens. 